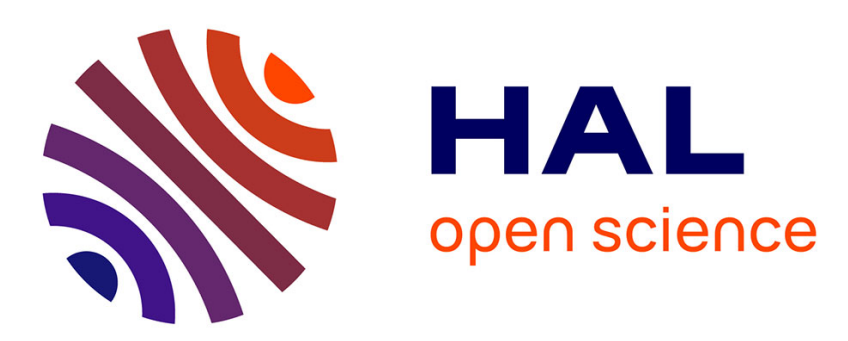

\title{
Défaire et refaire un groupe à risque. Objectivation et prévention du sida chez les homosexuels masculins à l'ère des antirétroviraux
}

\author{
Mathieu Trachman, Gabriel Girard, Maud Gelly
}

\section{- To cite this version:}

Mathieu Trachman, Gabriel Girard, Maud Gelly. Défaire et refaire un groupe à risque. Objectivation et prévention du sida chez les homosexuels masculins à l'ère des antirétroviraux. Population (édition française), 2018, 73 (4), pp.787-807. 10.3917/popu.1804.0787 . hal-02179479

\author{
HAL Id: hal-02179479 \\ https://hal.science/hal-02179479
}

Submitted on 10 Jul 2019

HAL is a multi-disciplinary open access archive for the deposit and dissemination of scientific research documents, whether they are published or not. The documents may come from teaching and research institutions in France or abroad, or from public or private research centers.
L'archive ouverte pluridisciplinaire $\mathbf{H A L}$, est destinée au dépôt et à la diffusion de documents scientifiques de niveau recherche, publiés ou non, émanant des établissements d'enseignement et de recherche français ou étrangers, des laboratoires publics ou privés. 


\title{
Défaire et refaire un groupe à risque. Objectivation et prévention du sida chez les homosexuels masculins à l'ère des antirétroviraux
}

\begin{abstract}
En France, chaque année, plusieurs milliers de personnes découvrent leur séropositivité, bien que le travail d'information et de prévention se soit poursuivi sans relâche depuis les années 1990. Le rôle des associations et des pouvoirs publics a été déterminant dans la lutte contre l'épidémie. Les auteur.e.s reviennent sur l'histoire des politiques de santé publique contre le sida et sur les conditions du passage d'une politique de prévention universelle à une politique ciblée sur les personnes les plus exposées. Pour ce faire, et en s'appuyant sur différentes données, ils analysent les réponses apportées à trois questions cruciales: qu'est-ce qu'un groupe à risque? Comment cibler les personnes à risque sans les stigmatiser? Quelles stratégies permettent de les protéger?
\end{abstract}

En France, depuis le début de l'épidémie de sida, la définition des frontières du risque fait problème: dans un souci d'éviter toute stigmatisation, la culture de la santé publique hésite entre l'attribution du risque à des groupes ou à des comportements (Calvez, 2004; Pollak, 1988). Dans les années 1980 et 1990, la lutte contre le sida est devenue une cause concernant l'ensemble de la population, ce qui a permis la mise en place d'une politique de santé publique sans désigner des populations spécifiques, ou du moins sans le faire explicitement (Dodier, 2003; Pinell, 2002). À partir du cas des immigrés, Didier Fassin a analysé les problèmes de ce type de politique et montré qu'elle reposait sur un régime de production des savoirs fondé sur l'évitement et la surdétermination (Fassin, 1999) : le refus de stigmatiser conduit les médecins ou les politiques

\footnotetext{
* Ined, Iris / Ehess, France.

** CRESPPA-CSU, AP-HP, France.

*** École de santé publique, Université de Montréal, Canada.

Correspondance: Mathieu Trachman, Ined, 133 Bd Davout, 75980 Paris cedex 20. mathieu.trachman@ined.fr
} 
à ne pas constituer les appartenances sexuelles ou ethniques en variables explicatives, celles-ci persistant pourtant dans les discours sans être questionnées.

À la fin des années 2000, ce régime de production et de diffusion des savoirs, oscillant entre l'indicible et l'impensé, est révolu. Comme l'illustre le rapport Lert-Pialoux (Lert et Pialoux, 2010) sur la réduction des risques, les experts de la prévention identifient de plus en plus explicitement des « groupes à haut risque ${ }^{(1)}$. Sur la base d'outils statistiques, dans une volonté d'efficacité et de réalisme, l'épidémiologie comme le monde associatif s'accordent désormais pour définir comme principaux groupes exposés au VIH les homosexuels masculins et les personnes immigrées d'Afrique subsaharienne. Témoin des enjeux d'efficacité thérapeutique qui traversent la définition des populations, ces deux groupes les plus exposés sont souvent nommés « groupes de transmission » dans la littérature épidémiologique puis, plus récemment, « populations-clés » (Desgrées du Loû et Spire, 2013), notion utilisée par l'Organisation mondiale de la santé et l'Onusida. Ces constats permettent la mise en place de programmes spécifiques, mais non perçus comme discriminatoires. Comment comprendre cette évolution? Pour une partie des acteurs de la lutte contre l'épidémie, au silence des années 1980 aurait succédé un réalisme scientifiquement fondé et plus efficace. Ce processus d'explicitation de ce qui ne pouvait être dit permettrait d'écarter tout enjeu politique ou moral au profit des seuls faits épidémiologiques.

Cette lecture occulte cependant les évolutions de l'objectivation des groupes à risque et les enjeux politiques et moraux qui y restent associés. À la suite des études sociologiques sur la quantification, nous abordons le travail d'objectivation comme producteur de populations cibles, dans un double mouvement de « visée de réalité » et d'« institution » de ces populations (Desrosières, 2008, chap. 11). Dans cette perspective, l'identification d'un groupe à risque doit tenir compte de plusieurs contraintes : tout d'abord, se donner les moyens de saisir les évolutions des comportements à risque et les rapports au risque en mettant en place des dispositifs de collecte spécifiques; ensuite circonscrire des populations qui donnent prise au travail de prévention; délimiter des espaces où les groupes ainsi définis sont concentrés et/ou moins difficiles à atteindre; identifier des acteurs intermédiaires susceptibles d'atteindre les groupes recherchés pour y mener un travail de prévention; et enfin tenir compte des enjeux sociaux et moraux, notamment en termes de stigmatisation que la production de données sur ces populations implique (Schiltz, 2005).

Dans le cas du VIH, la définition des groupes à risque a connu ces dernières années des évolutions importantes, en particulier du fait de l'efficacité des traitements antirétroviraux. Ces traitements réduisent la mortalité liée au sida,

(1) Commandé par la Direction générale de la santé en 2009, la rédaction du rapport est confiée à France Lert, directrice de recherche, épidémiologiste à l'Inserm, et Gilles Pialoux, chef de service de maladies infectieuses à l'Hôpital Tenon à Paris. 
mais ils permettent également de réduire le risque de transmission. On considère ainsi que, sous certaines conditions, un séropositif sous traitement dont la charge virale est indétectable ne peut pas transmettre le VIH (Race, 2001; Vernazza et al., 2008). Dès lors, ni les pratiques sexuelles ni le statut sérologique ne sont des informations suffisantes pour définir une prise de risque, celle-ci étant aussi dépendante de la mise sous traitement des personnes atteintes et de son observance. De plus, la situation épidémiologique est jugée préoccupante : les nouvelles infections se maintiennent en France à un niveau élevé (environ 6000 par an selon Cazein et al., 2014); le nombre de cas de syphilis récents augmente depuis 2000, et a doublé chez les hommes homo-bisexuels entre 2007 et 2014 (de 500 à 1000 cas par an selon Ndeikoundam Ngangro et al., 2016); l'incidence du VIH chez les hommes ayant des rapports sexuels avec des hommes est 200 fois plus élevée que chez les hétérosexuels (Le Vu et al., 2010). Les cibles des interventions de prévention doivent donc être redéfinies. Depuis les années 2000, les personnes vivant avec le VIH mais ignorant leur statut ou les séro négatifs ayant des pratiques à haut risque d'exposition au virus sont au cour des préoccupations de la santé publique. Dans ce contexte, les enquêtes épidémiologiques et sociocomportementales ciblent des espaces et des groupes de plus en plus précis. Cette évolution détermine enfin de nouvelles pratiques de prévention, en particulier le dépistage rapide, notamment « ciblé », auquel des intervenants associatifs, et non plus seulement des médecins, sont désormais habilités à participer. Se développe aussi la prophylaxie préexposition, qui repose sur un usage préventif et non curatif des traitements antirétroviraux qui peuvent être utilisés par des personnes non séropositives. Le dépistage répété comme la prophylaxie préexposition n'ont à aucun moment été envisagés pour l'ensemble de la population. Ces deux interventions contribuent à refaire un " groupe à risque », en prolongent et précisent la définition savante.

L'usage de la notion de risque elle-même évolue. L'accent mis dans les années 1990 sur les « prises de risque », c'est-à-dire sur une exposition ponctuelle au risque de contamination par le VIH par un rapport sexuel sans préservatif, et sur les « comportements à risque » qui supposent des prises de risques répétées, avait pour objectif de distinguer les personnes des comportements qu'elles adoptent à une période de leur vie, et ainsi de défaire les « groupes à risque », notion jugée stigmatisante. La diffusion d'informations, d'abord dans l'espace associatif puis par des agences d'État à la fin des années 2000, sur les niveaux de risque de contamination associés à diverses pratiques sexuelles, prend acte de la difficulté à utiliser des préservatifs de façon constante et prolongée. Il s'agit alors d'outiller les personnes de connaissances leur permettant de mettre en œuvre des pratiques de « réduction des risques », par exemple en remplaçant une pratique «à haut risque » de contamination, telle que la pénétration anale sans préservatif par un partenaire de statut sérologique inconnu, par une pratique à moindre risque de contamination, telle que la fellation sans préservatif. De plus, indépendamment des connaissances sur les niveaux de risque de contamination, les rapports au risque VIH 
chez les hommes homosexuels engagent la perception du risque par l'individu, son expérience ordinaire de la prévention, sa conception des relations sociales entre homosexuels (Girard, 2013).

Cet article retrace l'évolution du travail d'objectivation des groupes à risque et du travail de prévention qui leur est spécifiquement destiné. Larticle repose sur des matériaux disparates qui ont pour objectif de saisir une logique générale à travers la diversité des objets ${ }^{(2)}$. Il s'appuie d'abord sur l'analyse des enquêtes produites sur les homosexuels masculins et le sida en France depuis les années 1990. Pour saisir l'objectivation en pratique, l'article mobilise également des enquêtes de terrain sur la mise en œuvre du dépistage rapide du VIH et sur la prophylaxie préexposition. Les données sur le dépistage s'appuient sur le dépouillement des archives de l'association Aides et des rapports des administrations en charge de la santé publique ${ }^{(3)}$. Les données concernant la prophylaxie préexposition sont issues de notre participation, en tant que sociologues, à l'essai Ipergay, dont l'objectif était d'évaluer l'efficacité et la faisabilité de la prophylaxie préexposition $^{(4)}$ : nous avons réalisé des entretiens avec les personnes impliquées dans l'essai (participants, promoteurs, salariés d'association ou de l'Agence nationale de recherche sur le sida et les hépatites virales, ANRS), participé à des réunions, recueilli de la documentation et des supports de communication produits dans le cadre de la campagne, et assuré une veille des débats dans les espaces communautaires.

\section{Comment s'établit le relâchement de la protection? Identification de nouveaux comportements à risque et évolutions du travail d'objectivation}

La mise en place d'une politique de lutte contre le sida en France a favorisé la production de savoirs médicaux, épidémiologiques mais aussi sociocomportementaux, notamment sociologiques et anthropologiques, conçus comme des outils indispensables à lintervention. L'ANRS a joué ici un rôle essentiel comme agence de financement, d'organisation et de centralisation des savoirs sur le VIH (Calvez, 2004). Au début des années 2000, un consensus s'établit pour reconnaître un changement dans les pratiques de prévention : « nouvelles réalités, nouveaux enjeux » des liens entre sida et immigration (ANRS, 2002), " nouveau rapport au risque » des homosexuels masculins (Bozon et Doré, 2007). Pour les gays, des enquêtes établissent peu à peu l'idée d'un relâchement des pratiques protégées ou relapse : après une période marquée par l'adoption

(2) Le projet collectif articulant les recherches des auteurs de l'article, dont ce texte est issu, a reçu un financement par l'ANRS.

(3) Ce travail de recherche documentaire est articulé, dans la thèse de sociologie de Maud Gelly (Gelly, 2016a, 2016b, 2018) qui a bénéficié d'un financement partiel par Sidaction, à une enquête ethnographique par entretiens avec des agents du dépistage et par observation directe des actions de dépistage.

(4) Gabriel Girard a été membre du conseil scientifique de l'essai de 2010 à 2016. 
massive du préservatif dans la sexualité entre hommes, son usage est en recul. Ces changements de comportements ne doivent pas occulter ceux de leurs instruments de mesure, ni des populations qu'ils ciblent.

\section{De nouvelles populations cibles}

Depuis la fin des années 1990, de nombreuses études quantitatives ont établi l'augmentation des comportements sexuels à risque chez les gays. Cette tendance est concordante avec des enquêtes comparables menées dans d'autres pays occidentaux (Australie, Canada, États-Unis). En France, l'enquête Presse Gay ${ }^{(5)} 2000$ met ainsi en évidence une augmentation de la déclaration des pratiques de pénétration anale non protégée avec un partenaire occasionnel depuis la précédente enquête (1997), couplée avec une utilisation moins fréquente du préservatif avec le partenaire stable, et une recrudescence des maladies sexuellement transmissibles autres que le VIH : en 2000, $23 \%$ des répondants déclarent des pénétrations anales non protégées avec des partenaires occasionnels, alors qu'ils étaient $16 \%$ en 1997. Concernant les maladies sexuellement transmissibles (MST), $13 \%$ des répondants déclaraient avoir été infectés au cours de l'année en 1997 et 15,7 \% en 2000 (Adam et al., 2001). Les évolutions des comportements à risque depuis 2000 sont présentées comme un « relâchement » de la vigilance préventive. Ce recul des pratiques préventives est confirmé au cours des 14 années qui suivent dans différentes enquêtes (Baromètre Gay en 2002; Enquête Presse Gay en 2004; Baromètre Gay en 2005; Prevagay en 2009; Enquête Presse Gaie et Net Gay Baromètre en 2011). Ces enquêtes reposent sur des échantillons de convenance et des stratégies de collecte différentes, qui expliquent sans doute les variations des résultats : selon les enquêtes, entre $20 \%$ et $40 \%$ des homosexuels sont considérés comme ayant des pratiques à risque pour la transmission du VIH.

De plus, les populations que visent les enquêtes précédemment citées ne sont pas tout à fait les mêmes. Dans les enquêtes des années 1990, un consensus s'établit pour faire des hommes ayant des relations sexuelles avec des hommes (HSH) la population cible. En saisissant les personnes par leurs pratiques, cette catégorie a pour objectif de centrer le travail sur les pratiques sexuelles et d'écarter les questions d'identification, complexes dans un contexte de stigmatisation et supposées secondaires dans la dynamique de l'épidémie ${ }^{(6)}$. Ainsi les hommes qui ont des rapports sexuels avec des hommes, mais qui ne se déclarent pas ou ne se considèrent pas comme gais, et les hommes qui ont des rapports sexuels avec des partenaires des deux sexes ne sont pas exclus. Plus généralement, les enquêtes Presse Gay se donnaient initialement pour objectif

(5) Mises en place à partir de 1985, ces enquêtes sont ainsi nommées parce que les questionnaires étaient diffusés via la presse à destination des homosexuels masculins; 14 éditions ont été réalisées, et depuis 2004 l'enquête est également diffusée sur des sites internet à destination des homosexuels masculins.

(6) Pour une analyse critique de la catégorie de HSH et de sa migration de l'épidémiologie vers l'espace associatif, voir Thomas (2011). 
de saisir une diversité de profils, même si le mode de recrutement, par voie de presse, touchait plutôt des gays qui se définissent comme tels et ont un certain sentiment d'appartenance à une communauté. Si cet objectif n'a pas disparu, depuis 2000 plusieurs enquêtes ciblent des populations plus circonscrites : celles qui fréquentent les lieux de sociabilité sexuelle et les sites de rencontre bareback $^{(7)}$, les multipartenaires, les jeunes ou les séropositifs, populations considérées comme cibles pour les discours de prévention.

Ces populations sont saisies à travers des espaces de plus en plus nettement circonscrits, en particulier les commerces gays. Ce n'est pas seulement l'espace des enquêtes, c'est aussi le regard sur ces espaces qui évolue. Les lieux de sociabilité sexuelle gaie ne sont pas absents des recherches initiées par l'ANRS au cours des années 1990 : entre 1993 et 1997, une série d'enquêtes ethnographiques est menée dans les lieux de drague, qu'ils soient extérieurs ou commerciaux (de Busscher et al., 1999; Mendès-Leite et al., 2000). Ces recherches-actions ont pour objectif de favoriser la prévention dans ces espaces : les backrooms (des espaces de rencontre sexuelle dans les arrière-salles ou les sous-sols des bars gais) y apparaissent comme un espace où le risque peut être négocié : « une fois écartés certains fantasmes prudes sur les dangers de la promiscuité' », elles peuvent être « des relais essentiels pour la prévention » (Mendès-Leite et de Busscher, 1997, p. 66).

À partir des années 2000, c'est une autre construction savante de ces espaces qui apparaît. Le Baromètre Gay 2000 est un sondage rapide qui cible les clients des « établissements gays parisiens » (bars, sex-clubs, saunas), faisant l'hypothèse qu'il s'agit là d'une " population particulièrement active sexuellement » (Adam, 2002). Le Baromètre Gay 2005 élargit le périmètre à l'ensemble du territoire français, et diffuse le questionnaire sur des lieux extérieurs de drague et des sites « identitaires » (Velter et al., 2005, 2006). L'enquête Prevagay 2009 (Velter et al., 2010) prolonge ce travail tout en se donnant les moyens d'objectiver la séroprévalence pour le VIH auprès de cette population : réalisée dans les bars, backrooms et saunas parisiens auprès de 886 hommes, l'enquête comportementale par questionnaire est complétée par un prélèvement sanguin (autoprélèvement capillaire au bout du doigt). L'appariement entre données comportementales et données biomédicales montre que 20 \% des gays séropositifs ignorent leur statut.

Au-delà des groupes et des espaces ciblés, ce sont les cadres d'interprétations des comportements sexuels des homosexuels masculins qui évoluent. Les travaux de Philippe Adam, responsable des Enquêtes Presse Gay 1997 et 2000 et du Baromètre Gay 2000, illustrent cette inflexion. Sa thèse de sociologie soutenue en 1997 analyse la constitution du monde des associations de lutte contre le sida en France, les trajectoires des militants et les évolutions des expériences homosexuelles depuis les années 1980 (Adam, 1997). Elle s'appuie

(7) La catégorie fait débat et saisit soit l'absence de protection, soit la volonté d'être contaminé, mais elle dénote toujours le refus du préservatif (Girard, 2013). 
sur des archives, des entretiens et les données des enquêtes Presse Gay. Celles-ci lui permettent de mettre en évidence la diversité des expériences sociosexuelles au principe de la gestion des risques, et soulignent l'importance de l'appartenance de classe et de la sociabilité homosexuelle pour expliquer les adaptations des conduites sexuelles dans un contexte de diffusion de l'épidémie. Le sida n'est pas alors conçu comme l'agent du changement, mais comme un révélateur d'évolutions antérieures, en particulier l'acceptation sociale croissante de l'homosexualité. De ce point de vue, le relâchement des pratiques préventives lui semble une explication réductrice d'une évolution plus générale des sexualités et des modes de vie gais (Adam et Schiltz, 1996).

À partir de la fin des années 1990, les recherches de P. Adam prennent une tournure plus psychosociologique, mais aussi plus alarmiste. Les résultats du Baromètre Gay 2000 le font conclure au développement d'une « culture du risque spécifique » chez les clients séropositifs des backrooms (Adam, 2002, p. 79). En 2004, il participe à une enquête auprès des usagers d'un site de rencontre français. L'enquête porte sur les désirs des gays, et pose notamment la question de l'émergence des fantasmes de non-protection. Les appartenances de classe ou les différences de sociabilité sont alors secondes au regard de critères plus proches de la santé publique : consommation d'alcool et de drogue, statut sérologique. Les différents modes de gestion des risques et l'influence des structures et des expériences sociales mises en avant dans la thèse de P. Adam laissent place à des comportements à risque, déterminés par de nouvelles techniques de rencontres. Enfin, la thèse mettait en évidence la place différentielle accordée à la sexualité selon les appartenances sociales : les conduites sexuelles étaient replacées dans les expériences non sexuelles des individus, et en particulier leur situation professionnelle et leur conception de l'homosexualité. Cette seconde enquête insiste plutôt sur la sexualité compulsive (sex drive), pulsion favorisée par les nouvelles technologies de rencontre sexuelle (sites de rencontre en ligne), mais indépendante des contextes sociaux (Adam et al., 2006, 2011).

Le ciblage de nouvelles populations implique donc la mise en place de dispositifs de collecte spécifiques, qui ont pour objectif de saisir certains homosexuels masculins. Ceux-ci ne sont pas seulement définis par leurs pratiques sexuelles, mais par la fréquentation de certains lieux, et plus généralement par un rapport à la sexualité spécifique dans lequel la prévention est secondaire.

\section{La redéfinition des pratiques à risque}

La redéfinition des pratiques à risque est une seconde évolution de ce travail d'objectivation. Au début des années 2000, le relâchement préventif est d'abord saisi par l'augmentation des déclarations de pénétrations anales non protégées (PANP), en particulier avec des partenaires occasionnels dont le statut sérologique est inconnu ou différent. Progressivement, dans les enquêtes suivantes, seules certaines PANP sont identifiées comme des indicateurs de 
risque : non pas celles qui ont lieu avec un partenaire stable, mais celles qui ont lieu avec des partenaires occasionnels; non pas une seule PANP au cours des 12 derniers mois, mais des PANP répétées. Il s'agit moins d'enregistrer des accidents qu'un rapport au risque.

La redéfinition des pratiques à risque est également liée à la mise en place du paradigme de la réduction des risques, qui s'impose en France au cours des années 2000 (Girard, 2016) : à la promotion du préservatif basée sur l'opposition entre pratiques à risque et pratiques safe, s'ajoute une échelle de risque qui prend en compte divers éléments tels le rôle sexuel (pénétration anale passive ou active), l'usage de lubrifiant ou le statut sérologique du partenaire. La réduction des risques cible les situations dans lesquelles le préservatif n'est pas utilisé. L'enquête Presse Gay et Lesbienne 2011 enregistre cette évolution : certes les pratiques anales non protégées et les fellations avec exposition au sperme y sont enregistrées comme des pratiques à risque, mais l'enquête enregistre également, comme l'édition de 2004, des pratiques associées à un risque de contamination non nul, mais moins élevé qu'une pénétration anale par un partenaire de statut sérologique inconnu. C'est le cas du serosorting (pratiquer la pénétration anale non protégée avec un partenaire du même statut sérologique). Dans un même ordre de pratiques associées à un niveau de risque variable, l'enquête interroge également le seropositionning (pratiquer la pénétration anale non protégée en position active ou passive, la première étant considérée comme moins risquée que la seconde) et le contrôle de la charge virale (Velter et al., 2013).

Cette redéfinition du risque a des conséquences sur les résultats de l'enquête de 2011 : dans celle-ci, 18,4\% des séropositifs et 58,5\% des séronégatifs déclarent se protéger systématiquement lors des pénétrations anales avec des partenaires occasionnels. Dans l'enquête de 2004, ils étaient respectivement $50,9 \%$ et $73,4 \%$. On peut faire l'hypothèse que cette évolution n'enregistre pas seulement une progression des PANP, mais une relativisation des risques qui y sont associés : une partie des enquêtés, et notamment les séropositifs, ont sans doute intégré l'idée que grâce aux traitements une PANP n'équivaut pas nécessairement à une pratique à risque, et peuvent être plus enclins à la déclarer. Le questionnaire lui-même, en interrogeant les diverses stratégies de réduction des risques, permet de mieux les faire connaître.

Les évolutions des comportements préventifs et le « relâchement » des homosexuels masculins au cours des années 2000 sont donc liés à une double redéfinition des populations ciblées par les enquêtes et les comportements à risque. Ce ne sont plus seulement les HSH, mais certains homosexuels masculins, certains espaces qui sont ciblés, dans un maillage plus fin de l'espace des pratiques sexuelles entre hommes. Ce ne sont plus seulement les pénétrations non protégées, mais certains comportements sexuels, qui sont la cible des enquêtes. Ainsi, ce ne sont pas seulement les comportements des homosexuels masculins qui évoluent, ce sont les instruments pour les mesurer qui changent. 


\section{L'ignorance et l'impuissance : le renouvellement des pratiques de prévention}

L'objectivation d'un relâchement des pratiques préventives chez les gays à partir de la fin des années 2000 conduit progressivement les agences de santé publique, et en particulier l'ANRS, à mettre en place de nouvelles stratégies de recherche, d'intervention et de prévention. Celles-ci prolongent le travail de définition des groupes à risque initié dans les enquêtes quantitatives. Ce travail n'engage plus des instruments de mesure, mais des pratiques de prévention dans lesquelles la définition d'une population cible est aussi celle d'une population sur laquelle il est possible d'intervenir. Ce travail suppose de produire une figure de l'individu à risque qui prenne acte de la non-protection, sans rendre vaine toute prévention. Deux interventions, le dépistage ciblé et la prophylaxie préexposition, illustrent cette tension. Pour la résorber, ce ne sont pas seulement les comportements sexuels qui vont être pris en compte, mais le rapport à la maladie et au risque VIH, caractérisé par l'ignorance de son statut sérologique et l'impuissance à mettre en œuvre une prévention sans faille. Dès lors que persistent des comportements sexuels à risque à l'ère des trithérapies, il importe de dépister et traiter précocement les individus qui se contaminent, et de proposer d'autres stratégies préventives aux individus qui n'utilisent pas systématiquement les préservatifs.

\section{Du dépistage renforcé au dépistage ciblé}

La reconnaissance de l'efficacité des trithérapies après 1996 est suivie au cours des années 2000 d'un redéploiement de l'action publique : le Conseil national du sida en 2006 et la Haute autorité de santé (HAS) en 2009, puis le Plan national de lutte contre le VIH publié en 2010, recommandent de renforcer le dépistage en ciblant les populations les plus exposées, notamment les gays. L'objectif est double: d'une part, réduire le nombre de diagnostics tardifs; d'autre part, dépister les 32000 à 68000 personnes (selon les estimations) qui ignorent leur séropositivité (Ministère de la Santé et des sports, 2010, p. 35-45). Autrement dit, il s'agit de trouver des « séropositifs qui s’ignorent ». Si le dépistage ciblé vise autant les hommes homosexuels que les personnes migrantes, sa spécificité en direction des premiers tient au fait qu'il est pensé comme un dépistage répété, afin de faire le diagnostic le plus rapidement possible après la contamination et de les soigner avant qu'ils ne contaminent leurs partenaires. Publiés en 2011, les résultats de l'essai HPTN052 confirment l'intérêt de la précocité du traitement en démontrant notamment que la transmission du VIH dans le couple s'en trouve réduite et que l'état clinique de la personne s'améliore (Cohen et al., 2011).

Si le renforcement du dépistage fait suite à la commercialisation des trithérapies en 1996, le ciblage du dépistage en direction des hommes homosexuels est plus récent, et s'opère dans le contexte de recrudescence des prises de risque 
et d'évolution des recommandations en faveur d'une mise sous traitement antirétroviral de plus en plus précoce, quel que soit le stade de l'infection.

\section{Renforcer le dépistage pour traiter les séropositifs et limiter les contaminations}

Dans les premières années de l'épidémie, la promotion du dépistage n'est pas au centre des stratégies de lutte contre le VIH : en l'absence de traitements efficaces, l'annonce de la séropositivité apparaît d'abord génératrice d'angoisse et d'isolement (Hirsch, 1991). Dans la seconde moitié des années 1980, les associations, les médecins spécialistes et les autorités de santé s'entendent pour refuser le dépistage obligatoire (Dodier, 2003). C'est après la commercialisation des trithérapies que le dépistage devient un axe majeur de l'action publique dans ce domaine, comme un moyen de repérer les « séropositifs qui s’ignorent », de les mettre rapidement sous traitement et donc de limiter la contamination. Le renforcement du dépistage, puis l'affirmation de son caractère « ciblé », vont alors faire l'objet d'un large consensus entre les agences de santé publique (comme la HAS et la Direction générale de la santé) et les associations.

Le Groupe national de recherche d'Aides a organisé le 22 février 1997 une journée thématique sur les « enjeux du dépistage ». Il en rend compte au Conseil national de l'association des 15 et 16 mars 1997 :

« Le groupe recommande au CN [Conseil National] de se déterminer pour une politique de dépistage plus incitative par la communauté vers la communauté [...] dans une perspective d'accès plus précoce aux traitements [...]. Si notre discours a été "il vaut mieux du counselling ${ }^{(8)}$ que du dépistage", il pourrait devenir "il vaut mieux un dépistage correctement proposé et correctement effectué qu'un (mauvais) counselling." "» ${ }^{(9)}$

La réorientation de l'association est rapide : les bénéfices des trithérapies sont immédiats et évidents, et ne font de doute pour aucun expert. L'association Aides se positionne même pour les « autoprélèvements, [...] envoyés au laboratoire, interprétés par un professionnel et donnés au patient par téléphone » car ils relèvent de la même « logique incitative ». Cette proposition n'aura pas de suite immédiate et la commercialisation des autotests arrivera près de vingt ans plus tard, en 2015.

Dans le même temps, le ministère de la Santé critique un dispositif de dépistage inégalement déployé en direction des publics les plus fragiles socialement, intervenant de façon trop tardive, et recommande un élargissement du dépistage ${ }^{(10)}$. Étendre le dépistage, c'est donc l'étendre dans le temps en le rendant plus précoce, et l'étendre dans l'espace social en direction de publics

(8) Méthode d'entretien recommandée entre autres pour le dépistage.

(9) Archives nationales, AN 20030474/3, Conseil national de AIDES, 15-16 mars 1997.

(10) Archives nationales, AN 20030474/50, AIDES Fédération/Actions thérapeutiques/Groupe national Information sur les traitements/Groupe de travail ministériel sur le dépistage précoce 1997, note de Françoise Bélingard-Deybach au directeur général de la santé, 25/03/1997. 
définis sur des critères non plus sexuels et comportementaux mais sociaux, comme en témoigne ce compte rendu de la réunion du 29 mai 1997 du sousgroupe information-communication du groupe de travail ministériel sur le diagnostic précoce de l'infection VIH :

« Deux grands enjeux sous-tendent le dépistage précoce : la prévention (réduction des risques de transmission aux partenaires) et la prise en charge (très précisément les traitements). [...] Les modèles de dépistage sous-jacent ramènent :

- au schéma classique du dépistage des maladies transmissibles hérité de [la] lutte contre les maladies sexuellement transmissibles ou de la lutte contre la tuberculose;

- au schéma des programmes de dépistage lié à la possibilité d'un bénéfice pour les individus et populations en termes de morbidité ou d'incapacités. Ce schéma devient possible.» ${ }^{(11)}$

La Division sida de la Direction générale de la santé (DGS) associe, comme c'est l'usage depuis les années 1990 dans le champ de la lutte contre le sida, l'État, les médecins et les associations, puisqu'y participent des représentants de la DGS, de l'Union régionale des médecins libéraux d'Île-de-France et les associations Arcat-Sida et Aides. L'élargissement du dépistage fait donc consensus entre l'État, le champ médical et l'espace associatif, de même que l'extension des publics ciblés par l'action publique. Mais le dépistage n'est pas élargi de façon indifférenciée dans l'espace social, et les hommes homosexuels vont faire l'objet d'un ciblage spécifique.

\section{Légitimer le dépistage ciblé}

En 2010, le ministère de la Santé rédige un plan national de lutte contre le $\mathrm{VIH} /$ sida et les infections sexuellement transmissibles (IST) pour la période 2010-2014 qui détermine cinq « axes stratégiques » de lutte contre l'épidémie. Le deuxième d'entre eux est le dépistage ${ }^{(12)}$. Lactivité de dépistage du VIH en France est alors stable depuis 2006 : 5 millions de tests sont pratiqués chaque année (soit 80 pour 1000 habitants), dont les trois quarts dans des laboratoires de ville et $7 \%$ en Centre de dépistage anonyme et gratuit (CDAG). La proportion de sérologies positives est de 2,2 \%o au niveau national et plus élevée en CDAG (3,6\%o; Cazein et al., 2014). Synthétisant les enquêtes réalisées sur cette question, le plan national identifie deux « profils » de retard au diagnostic : les « personnes appartenant à une population à risque identifiée » n'ayant pas fait de démarche de dépistage, n'ayant pas été en contact avec le système de soins ou n'ayant pas été dépistées lors de ce contact; et les « personnes à faible

(11) Archives nationales, AN 20030474/50, AIDES Fédération/Actions thérapeutiques/Groupe national Information sur les traitements/Groupe de travail ministériel sur le dépistage précoce 1997, compte rendu de la réunion du sous-groupe information-communication du groupe de travail ministériel sur le diagnostic précoce de l'infection au VIH de la Division Sida de la DGS, 29/05/1997.

(12) Le premier concerne la prévention, le troisième la prise en charge médicale, le quatrième la prise en charge sociale et la lutte contre les discriminations, le cinquième la recherche. 
risque «apparent» (personnes hétérosexuelles françaises par exemple) » (Ministère de la Santé et des sports, 2010, p. 35-45).

Le premier « profil » correspond à un groupe d'individus susceptibles de transmettre plus largement le VIH, pas le second. Si ces deux groupes ont un bénéfice individuel équivalent à être dépistés et traités précocement, la réduction du nombre de contaminations à l'échelon collectif est plus importante lorsque le dépistage cible le premier groupe. En d'autres termes, pour réduire la diffusion du virus à l'échelle de la population, il faut renforcer le dépistage parmi les hommes homosexuels et les personnes migrantes d'Afrique subsaharienne (les usagers de drogue ne représentent plus, depuis la mise en place des politiques d'échange de seringues et de substitution, que $1 \%$ des nouvelles contaminations).

Afin de dépister ces deux catégories d'individus, le plan national promeut un «trépied du dépistage ». Celui-ci comprend tout d'abord le dépistage de la population générale par une proposition de test plus systématique par les professionnels de santé de premier recours, notamment les médecins généralistes, ainsi que par l'ouverture en 2010 de la possibilité d'être dépisté sans prescription en laboratoire (article L. 6211-10 du Code de la santé publique). Le second volet est le « dépistage par les pairs pour les populations à forte incidence » : « il s'agit de développer une offre de dépistage dit communautaire, réalisé par les pairs. Cette modalité de dépistage vise à répondre aux besoins des populations qui ne veulent ou ne peuvent se rendre dans le dispositif « classique ». Cette modalité de dépistage s'adresse en premier lieu aux HSH ». Enfin le dépistage anonyme et gratuit réalisé par les CDAG, c'est-à-dire en réponse à une démarche volontaire des usagers (Ministère de la Santé et des sports, 2010, p. 35-45) complète le dispositif.

À chacune de ces deux logiques de l'action publique correspond un type d'acteur : les médecins généralistes pour mettre en ouvre le dépistage généralisé et que chaque individu dépisté y gagne une espérance de vie similaire à celle de la population générale; des dispositifs spécifiques pour mettre en œuvre le dépistage ciblé et que l'ensemble de la population y gagne un risque de contamination plus faible. Des dispositifs spécifiques, publics et associatifs, sont alors nécessaires pour atteindre ces populations cibles.

\section{Mobiliser les associations pour renforcer le dépistage des hommes homosexuels : l'émergence du dépistage communautaire}

Malgré le consensus scientifique et politique pour étendre le dépistage, l'action publique en la matière connaît peu d'évolution. Au début des années 2000 se multiplient, d'abord hors de France, des expériences de dépistage au sein de structures associatives menées avec l'utilisation de tests rapides, par prélèvement d'une goutte de sang au bout du doigt suivi d'une lecture immédiate du résultat du test. C'est le cas notamment du projet Checkpoint mené à Amsterdam depuis 2000 (Darmon, 2008). La plupart de ces expériences se 
veulent « communautaires », c'est-à-dire que le dépistage y est mis en œuvre non par des professionnels de santé, mais par des militants associatifs issus de la population cible, ici des hommes homosexuels.

En 2007, s'inspirant d'expériences associatives de ce type, la délégation départementale Aides de l'Hérault développe le projet Checkpoint de dépistage rapide, qui se veut un projet « de santé publique et de santé communautaire » ${ }^{(13)}$. Le conseil d'administration d'Aides des 24 et 25 février $2007^{(14)}$ engage l'association vers la validation par la recherche du dépistage « communautaire ». S'appuyant sur la recherche communautaire Com'Test ${ }^{(15)}$, Aides mène un travail de négociation avec la DGS et le ministère de la Santé, afin d'obtenir par un décret de novembre 2010 la légalisation de la pratique des tests rapides par des non-professionnels de santé(16).

En dépit de la « démédicalisation » affichée et de la tradition de critique du pouvoir médical dans laquelle se sont construites les associations de lutte contre le sida, la stratégie dans laquelle s'inscrit la prise en charge associative du dépistage met en évidence les évolutions de la division du travail de santé publique. La forme que prend cette participation associative est celle de la « recherche communautaire » menée, à l'instar des actions de santé communautaire (Fournier et al., 1995), « pour, par et avec» les groupes que la santé publique ne parvient pas à atteindre ni à connaître, comme l'explique un guide collectif sur la recherche communautaire rédigé par des militants, notamment d'Aides, et des chercheurs (Demange et al., 2012). La production de savoir apparaît donc étroitement liée à la délimitation de populations pour lesquelles ce savoir est valide. Il s'agit en fait d'un savoir pratique qui consiste à trouver les malades. L'extension du dépistage repose principalement sur des expériences de « dépistage communautaire » qui visent à atteindre, par l'intermédiaire de médiateurs (qu'ils soient salariés ou militants associatifs, ou plus rarement salariés d'institutions de soin), les groupes les plus exposés au VIH.

Avec les Enquêtes Presse Gay et Baromètres Gay, il s'agit de compter des hommes qui ne veulent ou ne peuvent se protéger du risque de contamination. Avec le dépistage ciblé, notamment « communautaire », il s'agit de les trouver le plus tôt possible après la contamination, et les premiers éléments d'évaluation de ce type de dépistage montrent une certaine efficacité du dispositif, puisque le taux de dépistage positif (8,6\%o) y est supérieur à celui des CDAG (Cazein et al., 2014). Avec la prophylaxie préexposition (PrEP), il s'agit de leur fournir les outils permettant de faire face à un risque qu'ils n’arrivent pas à contrôler.

(13) Aides, « Note sur l'ouverture d'un Checkpoint de dépistage à la délégation départementale de l'Hérault » (document préparatoire au CA des 8 et 9 septembre 2007), 2007.

(14) Aides, CA 24-25 février 2007, point 8, « Projet de résolution dépistage ».

(15) Recherche menée depuis 2009 par Aides pour évaluer le dépistage « communautaire », c'est-àdire mis en œuvre par des militants de l'association.

(16) « Arrêté du 9 novembre 2010 fixant les conditions de réalisation des tests rapides d'orientation diagnostique de l'infection à virus de l'immunodéficience humaine (VIH 1 et 2) », Journal Officiel de la République Française, 17 novembre 2010, n 0266, p. 20499. 


\section{La prophylaxie préexposition : prendre en charge les gays qui n'arrivent pas à se protéger}

L'essai de prophylaxie préexposition Ipergay (Intervention préventive de l'exposition aux risques avec et pour les gays), qui propose à des gays séronégatifs ayant des pratiques à risque d'évaluer l'efficacité d'un antirétroviral pour éviter la contamination, est un second exemple des nouvelles stratégies de prévention mises en ouvre au cours des années 2000 en France. Si les enjeux sociologiques de la prophylaxie préexposition sont nombreux (Auerbach et Hoppe, 2015), nous nous attachons ici à préciser la manière dont cet essai cible des individus particuliers. La littérature sur le sida a très tôt établi comment les représentations savantes de la sexualité, et en particulier de l'homosexualité masculine, contribuaient aux cadrages de la prévention, en opposant des bons et des mauvais sujets sexuels (Edelman, 2013, chap. 6; Treichler, 2013). En s'adressant aux gays qui prennent des risques sans avoir pour objectif premier de réformer leurs comportements, Ipergay ne met pas en avant l'exemplarité des gays qui ont recours au préservatif, mais la faillibilité de ceux qui n'y parviennent pas. Si cette image a pour ambition de cerner au plus près les caractéristiques des homosexuels qui prennent des risques, elle produit cependant une figure singulière, d'individus à la fois incapables de se protéger systématiquement et capables de suivre un traitement complexe, de rationaliser leur sexualité, et donc susceptibles d'être réceptifs à de nouvelles stratégies de prévention (Trachman et Girard, 2018).

Cette figure singulière distingue la prophylaxie préexposition (PrEP) d'un autre usage prophylactique des antirétroviraux, le traitement postexposition. Ce dernier est dispensé en France depuis 1998 (Paicheler, 2007) : les personnes ayant eu un rapport à risque peuvent prendre un traitement antirétroviral dans les heures qui suivent, et pendant 28 jours, pour limiter les probabilités d'infection. Malgré l'efficacité du traitement postexposition, peu d'individus y ont recours : l'objectif est alors de lutter contre un manque d'informations de la part des usagers potentiels comme des services médicaux (Lert et Pialoux, 2010). Dans le cas de la prophylaxie préexposition, ce n'est pas le manque de connaissances mais la difficulté des individus de mettre en place des pratiques préventives, et donc la nécessité de les renouveler, qui est au centre de l'attention des chercheurs. L'objectif n'est pas seulement de faire connaître, mais de donner aux individus des outils adaptés à leur rapport à la sexualité. C'est ce que montre l'essai Ipergay.

Ipergay débute en France en février 2012. C'est un essai randomisé de prophylaxie préexposition qui propose, sur la base du volontariat, à des gays séronégatifs ayant des pratiques à risque d'entrer dans un suivi de santé sexuelle renforcé, incluant la prise d'un antirétroviral, afin de limiter les risques de contamination. Seule une partie de l'échantillon, sélectionnée de manière 
aléatoire et en « double aveugle ${ }^{(17)}$, a accès au traitement; les autres prennent un placebo. Selon le protocole de l'essai, il s'agit de proposer « un schéma de prophylaxie pragmatique, simple à suivre ", dépendant de l'activité sexuelle du sujet. Concrètement, les participants doivent prendre un comprimé 24 heures avant un rapport sexuel (au plus tard 2 heures avant), puis un comprimé toutes les 24 heures pendant la période d'activité sexuelle, et enfin un dernier comprimé 24 heures après l'activité sexuelle. Selon les termes du protocole, c'est aussi la faisabilité de la PrEP sur une " population européenne bien informée » qui est évaluée, et donc les spécificités sociocomportementales de populations dans leur rapport aux risques et au sida. Par-delà son objectif d'évaluation de la PrEP, Ipergay favorise le recours à d'autres moyens de protection. La prophylaxie préexposition s'accompagne de la promotion d'une «prévention combinée » : tenir pour acquis qu'il y a des comportements à risque ne conduit pas à abandonner les modes de prévention comportementaux au profit d'une prévention biomédicale, mais à les renforcer.

Les critères d'inclusion de l'essai mêlent critères médicaux, démographiques et comportementaux : concernant ces derniers, sont visés des hommes (ou des personnes transgenres) de plus de 18 ans, ayant un « risque élevé de contamination par le VIH » (défini comme au moins deux PANP avec des partenaires différents dans les 6 derniers mois). Les sujets sont recrutés par des membres de l'association Aides et par d'autres associations, dans les lieux de sociabilités gays et les lieux de rencontres sexuelles, ainsi que sur les sites de rencontre sur internet. L'essai investit donc les nouveaux espaces du risque identifiés par les enquêtes au cours des années 2000.

Une campagne de communication est mise en place pour faciliter le recrutement de volontaires. Elle permet également de saisir la population ciblée par l'essai, au-delà des critères d'inclusion. La première campagne d'affiches mettait en scène des hommes aux profils relativement variés affirmant « Moi je suis Ipergay, et toi? ». L'énoncé semble redondant et peu informatif, mais fonctionne comme un procédé suscitant une interrogation.

L'essai cible également des « personnes à haut risque de contamination ${ }^{(18)}$. Diffusée en 2013, l'affiche affirmant que « Le VIH touche 200 fois plus les gays que les hétéros » n'est pas un simple rappel épidémiologique : elle rompt avec la prudence habituelle des campagnes de prévention qui, de crainte de stigmatiser, ne mettaient pas si nettement en avant les différences de prévalence. Cependant, la communication d'Ipergay n'oppose pas les individus responsables et les autres. Elle ne vise pas nécessairement des individus qui prennent intentionnellement des risques, comme dans la figure inquiétante qui hantait les débats autour du bareback (Girard, 2013). Dans les campagnes de prévention,

(17) C'est-à-dire que ni le participant ni le médecin ne savent ce que contiennent les comprimés (molécule active ou placebo).

(18) L'expression est employée dans la newsletter de l'été 2013 adressée aux médecins par l'équipe de coordination de l'essai. 
cette figure est présente une seule fois, dans un contexte très spécifique : la campagne destinée à un site internet spécialisé, qui se réfère explicitement à la sous-culture du bareback. Dans ce cas, c'est « l'amour »du « sexe sans capote » qui est mis en avant, même si le texte d'information insiste immédiatement sur la prévention combinée. La prévention se fait donc dans une « totale acceptation des pratiques », expression plusieurs fois reprise dans les campagnes.

Ce discours est exceptionnel. Ce sont plutôt ceux qui sont impuissants à se protéger de manière continue, qui ne rejettent pas la prévention mais pour qui elle est problématique, qui sont ciblés par les campagnes. Un bandeau diffusé sur internet montre un jeune homme interrogatif, et demande : « Pour te protéger du VIH, tu fais quoi? ». Une affiche de 2013 demande si ses lecteurs ont « un problème avec le préservatif ». La première page d'un dépliant pour recruter des volontaires montre un homme fixant l'objectif, accompagné d'une question : "Vous en avez assez d'angoisser après chaque plan? ». Faire face aux difficultés de la prévention et mobiliser les affects est au centre de cette campagne.

L'essai ne saisit donc pas un sujet qui fait ce qu'il veut, que ce soit en se protégeant ou en ne se protégeant pas. L'engagement dans l'essai suppose que les volontaires veuillent se réformer sans y parvenir; il prend acte de pratiques à risque en les lisant comme une impuissance à se protéger et non une volonté de ne pas se protéger. Le ciblage des populations à l'œuvre dans Ipergay prend ainsi sens au regard du «relâchement » des pratiques préventives : les gays qui prennent des risques sont considérés comme des individus faillibles et non des « irrécupérables » ou des « inconscients », qui prennent volontairement des risques et ne comptent pas ne plus en prendre. La reconnaissance d'une faiblesse de certains gays face au risque donne au contraire une prise pour une prévention qui prend acte de l'insuffisance de la prévention comportementale.

\section{Conclusion}

Au cours des années 2000, par le croisement d'enquêtes répétées, de données épidémiologiques et de dispositifs de prévention spécifiques, des savoirs et des interventions définissent une population d'homosexuels masculins considérés comme des cibles privilégiées pour la prévention. Si prévenir la stigmatisation n'est plus déterminant, la fabrique d'un groupe à risque n'a pas pour seul principe le réalisme vis-à-vis des rapports au risque. Quelques années avant la mise sur le marché des trithérapies, Michaël Pollak écrivait à propos des débats entre politique préventive généraliste ou ciblée :

« si l'on parle souvent d'un nécessaire ajustement des messages aux publics ciblés, il convient par ailleurs de distinguer entre les deux définitions de l'ajusté, orientées l'une vers la justesse (technique), l'autre vers la justice (politique et civique) »(Pollak, 1988 p. 20). 
Au début des années 2000, la justesse technique l'emporte sur la justice politique, la crainte de stigmatiser un groupe s'estompe, et les techniques d'objectivation et de prévention sont renouvelées et ajustées au groupe ciblé. Cette justesse technique n'est cependant pas sans impensés, qui relèvent différents aspects de la définition d'un groupe à risque : des pratiques, mais aussi des espaces où l'on peut saisir ce groupe, et la détermination de certaines caractéristiques sociodémographiques des individus (c'est-à-dire que d'autres caractéristiques sociodémographiques sont jugées non pertinentes pour la définition du groupe).

Au-delà du constat de la recrudescence des prises de risque, c'est la définition des rapports au risque et à la séropositivité qui évoluent. La reconnaissance d'un "relâchement » de la prévention chez les gays fait naître un nouveau problème, déterminant pour la fabrication d'un groupe à risque : quelles prises se donner sur ces populations qui semblent refuser la prévention telle qu'elle avait été pratiquée jusque-là? Comme dans la "médecine de surveillance » décrite par David Armstrong dans sa généalogie du dépistage au cours du $\mathrm{XX}^{\mathrm{e}}$ siècle (Armstrong, 2012), la cible n'est pas le consultant mais le non-consultant, celui qui échappe aux mailles des dispositifs de prévention. La mobilisation d'acteurs associatifs conçus comme les membres de la population cible est un élément permettant d'atteindre cet objectif. L'enjeu n'est pas seulement de concevoir des nouveaux moyens de saisir cette population, mais de produire une image des individus preneurs de risque qui reconnaît la non-protection comme une possibilité, sans rendre caduque toute intervention et toute prévention. Il s'agit donc d'ajuster les groupes ciblés aux techniques de prévention. La prise en compte de la complexité des rapports au risque et à la sexualité, caractérisés par l'ignorance et l'impuissance, peut être ainsi comprise à la fois comme une description plus fine des prises de risque et comme la délimitation d'une cible pour le travail de prévention. La définition d'un groupe à risque ne s'appuie pas uniquement sur des données médicales et épidémiologiques dont elle accompagnerait les évolutions, elle est prise dans un ensemble de contraintes qui sont celles de la modernité thérapeutique analysée par Nicolas Dodier : développement des essais contrôlés et randomisés, mise à distance du paternalisme, mobilisation d'associations, de comités d'éthique et de profanes (Dodier, 2003). Le cas des homosexuels masculins montre que la définition d'un groupe à risque doit également justifier la prévention en offrant des prises pour son action. 


\section{RÉFÉRENCES}

ADAM Philippe, 1997, « Expérience intime et action collective. Sexualité, maladie et lutte contre le sida », Thèse de sociologie, Paris, EHESS.

ADAM Philippe, 2002, «Baromètre Gay 2000 : résultats du premier sondage auprès des établissements gays parisiens ", Bulletin épidémiologique hebdomadaire, $\mathrm{n}^{\circ} 18$, p. 77-80.

ADAM Philippe, SCHILTz Marie-Ange, 1996, « Relapse et cantonnement du risque aux marges de la 'communauté' : deux idées reçues à l'épreuve de l'enquête presse gaie », in Calvez Marcel, Schiltz Marie-Ange, Souteyrand Yves (dir.), Les homosexuels face au sida. Rationalités et gestions des risques, Paris, ANRS.

ADAM Philippe, HAUET Eric, CARON Caroline, 2001, «Recrudescence des prises de risque et des MST parmi les gays : résultats préliminaires de l'Enquête presse gay $2000 »$, Paris, Rapport InVS, ANRS-DGS.

ADAM Philippe, MURPhy Dean, DE WIT John, 2011, « When do online sexual fantasies become reality? The contribution of erotic chatting via the Internet to sexual risk-taking in gay and other men who have sex with men », Health Education Research, 26(3), p. 506-515.

Adam Philippe, De Wit John, Alexandre Antonio, Paolucci C., Troussier Thierry, 2006, «Les effets de la dépression sur l'activité sexuelle et la prise de risques parmi les gays français utilisateurs de sites de rencontre sur Internet », Sexologies, 15(3), p. 176-182.

ARMSTRONG David, 2012, « Screening: Mapping medicine's temporal spaces », Sociology of Health and Illness, 34(2), p. 177-193.

AuerbaCH Judith D., Hoppe Trevor A., 2015, « Beyond 'getting drugs into bodies': social science perspectives on pre-exposure prophylaxis for HIV », Journal of the International AIDS Society, 18(3), p. 1-5.

ANRS, 2002, Sida, immigration et inégalités : nouvelles réalités, nouveaux enjeux, Paris, ANRS.

BOzOn Michel, DORÉ Véronique (dir.), 2007, Sexualité, relations et prévention chez les homosexuels masculins. Un nouveau rapport au risque, Paris, ANRS.

De BussCher Pierre-Olivier, MendĖS-LeIte Rommel, Proth Bruno, 1999, « Lieux de rencontres et backrooms", Actes de la recherche en sciences sociales, 128(1), p. 24-28.

Calvez Marcel, 2004, La prévention du sida: les sciences sociales et la définition des risques, Rennes, PUR.

CAZEIn Françoise, LE STRAT Yann, SARR Aminata, RAmus Charly, BouCHE Nadine, LE VU Stéphane, 2014, « Dépistage de l'infection par le VIH en France, 2003-2013 », Bulletin épidémiologique hebdomadaire, n³2-33, p. 234-340.

COHEN Myron, THE HPTN 052 STUdy TEAM, 2011, « Prevention of HIV-1 infection with early antiretroviral therapy ", New England Journal of Medicine, 365(6), p. 493-505.

DARMON Laetitia, 2008, « Tests rapides. La France lambine », Le Journal du sida, n' 202, p. 29-30. 
Demange Élise, Henry Émilie, BeKelynCK Anne, PrÉAu Marie, 2012, « Petite(s) histoire(s) de la recherche communautaire », in Demange Élise, Henry Émilie, Préau Marie, De la recherche en collaboration à la recherche communautaire. Un guide méthodologique, Paris, ANRS/Coalition Plus, Collection Sciences sociales et sida, p. 15-24.

DESGRÉES Du LOÛ Annabel, SPIRE Bruno, 2013, «Éditorial. Des études essentielles pour adapter la prévention et la prise en charge des populations clés », Bulletin épidémiologique hebdomadaire, $\mathrm{n}^{\circ}$ 39-40, p. 494-495.

DESROSIÈRES Alain, 2008, Gouverner par les nombres : L'argument statistique II, Paris, Presses de l'École des mines.

DODIER Nicolas, 2003, Leçons politiques de l'épidémie de sida, Paris, EHESS.

Edelman Lee, 2013, L'impossible homosexuel. Huit essais de théorie queer, Paris, Epel.

FASSIN Didier, 1999, «L'indicible et l'impensé : la question 'immigrée' dans les politiques du sida », Sciences sociales et santé, 17(4), p. 5-36.

FOURNIER Pierre, POTVIN Louise, 1995, « Participation communautaire et programmes de santé : les fondements du dogme », Sciences sociales et santé, 13(2), p. 39-59.

GELlY Maud, 2016a, «Classer pour soigner? Savoirs d'État, idéologie préventive, raisons pratiques. Enquête sociologique sur le dépistage du sida », Thèse de sociologie, Paris, Université Paris VIII.

GELLY Maud, 2016b, «Inégalités en tous genres face au décès par sida », Agone, n 58, p. 135-150.

GELLY Maud, 2018, «Le classement des publics aux guichets de la santé : raisons pratiques et travail discrétionnaire », Sociologie du travail, 60(1).

GIRARD Gabriel, 2013, Les homosexuels et le risque du sida. Individu, communauté et prévention, Rennes, PUR.

GIRARD Gabriel, 2014, « Réduire les risques. Homosexualité, prévention du VIH et controverses associatives », Politix, 27(108), p. 99-119

HiRSCH Emmanuel, 1991, AIDES. Solidaires, Paris, Le Cerf.

LE Vu Stéphane, LE Strat Yann, BARIn Francis, Pillonel Josiane, CAZEIN Françoise, BOUSQUET Vanina et al., 2010, «Population-based HIV-1 incidence in France, 2003-08: A modelling analysis », The Lancet Infectious Disease, 10(10), p. $682-687$

LERT France, PiAlOUX Gilles, 2010, « Prévention et réduction des risques dans les groupes à haut risque vis-à-vis du VIH et des IST », Paris, Rapport de la mission RDRS auprès de la Direction générale de santé.

MENDÈS-LEITE Rommel, DE BUSSCHER Pierre-Olivier, 1997, Back-rooms. Microgéographie « sexographique » de deux back-rooms parisiennes, Lille, Question de Genre/GKC.

MENDĖS-LEITE Rommel, PROTH Bruno, DE BUSSCHER Pierre-Olivier, 2000, Chroniques socio-anthropologiques au temps du SIDA : trois essais sur les (homo)sexualités masculines, Paris, L'Harmattan.

MINISTÈRE DE LA SANTÉ ET DES SPORTS, 2010, Plan national de lutte contre le VIH/sida et les IST 2010-2014.

NDEIKOUNDAM NGANGRO Ndeindo, VIRIOT Delphine, FOURnET Nelly, DE BARBEYRAC Bertille, GOUBARD Agathe, DUPIN Nicolas, JANIER Michel, et al., 2016, « Les infections sexuellement transmissibles bactériennes en France : situation en 2015 et évolutions récentes », Bulletin épidémiologique hebdomadaire, n 41-42, p. 738-744.

ONUSIDA, 2014, Accélérer la riposte. Mettre fin à l'épidémie de sida d'ici à 2030.

PAICHELER Geneviève, 2007, « La gestion d'un risque insaisissable entre expertise scientifique et vécu intime : la prophylaxie des accidents d'exposition sexuelle au VIH », Sociologies et sociétés, 39(1), p. 197-216. 
Pinell Patrice (dir.), 2002, Une épidémie politique. La lutte contre le sida en France, (1981-1996), Paris, PUF.

POllak Michaël, 1988, Les homosexuels et le sida. Sociologie d'une épidémie, Paris, Métailié.

RACE Kane, 2001, « The indetectable crisis: Changing technologies of risk », Sexualities, 4(2), p. 167-189.

SCHILTZ Marie-Ange, 2005, «Faire et défaire des groupes : l'information chiffrée sur les "populations difficiles à atteindre" », Bulletin de méthodologie sociologique, $\mathrm{n}^{\circ} 86$, p. 30-54.

THOMAS Gaëtan, 2011, « Définir pour compter », Revue monstre, n 3, p. 38-42.

TRACHMAN Mathieu, GIRARD Gabriel, 2018, « Targeting fallible men: Communication strategies and moral issues in a pre-exposure prophylaxis trial ", Journal of AIDS, $\mathrm{n}^{\circ} 79$, p.S13-S19

TREICHLER Paula, 2013 [1987], « Le sida, l'homophobie et le discours biomédical : une épidémie de signification », Genre, sexualité et société, n 9.

Velter Annie, Michel Alice, Semaille Caroline, 2005, Baromètre Gay 2002, SaintMaurice, InVS.

Velter Annie, Saboni Leila, Bouyssou Alice, Semaille Caroline, 2013, «Comportements sexuels entre hommes à l'ère de la prévention combinée - résultats de l'enquête Presse gays et lesbiennes 2011 », Bulletin épidémiologique hebdomadaire, n०39-40, p. 510-516.

Velter Annie, Michel Alice, Pillonel Josiane, JaCQuier Guy, SEMAille Caroline, 2006, « Baromètre Gay 2005 : enquête auprès des hommes fréquentant les lieux de rencontre gay franciliens », Bulletin épidémiologique hebdomadaire, $\mathrm{n}^{\circ} 25$, p. 178-180.

Velter Annie, BARIN Francis, BOUYSSOU Alice, LE Vu Stéphane, GuINARD Jérôme, Pillonel Josiane, SEMAille Caroline, 2010, « Prévalence du VIH et comportement de dépistage des hommes fréquentant les lieux de convivialité gay parisiens, Prevagay 2009 », Bulletin épidémiologique hebdomadaire, n 45-46, p. 464-466.

Vernazza Pietro, Hirschel Bernard, Bernasconi Enos, Flepp Markus, 2008, « Les personnes séropositives ne souffrant d'aucune MST et suivant un traitement antirétroviral efficace ne transmettent pas le VIH par voie sexuelle », Bulletin des médecins suisses, 89(5), p. 165-169. 


\section{Mathieu TrACHMAN, Maud Gelly, Gabriel GIRARD • DÉfAIRE ET REFAIRE UN GROUPE À RISQUE. OBJECTIVATION ET PRÉVENTION DU SIDA CHEZ LES HOMOSEXUELS MASCULINS À L'ÈRE DES ANTIRÉTROVIRAUX}

Depuis les années 2000, les politiques de lutte contre le VIH/sida en France ont connu une évolution importante. Au cours des années 1990, la lutte contre le VIH est devenue une cause nationale dont les actes de prévention s'adressent à l'ensemble de la population afin de prévenir toute stigmatisation. Dans les années 2000, la reconnaissance de l'existence de " groupes à risque " n'a plus posé problème et a déterminé des actions plus ciblées. Constatant la recrudescence des prises de risques, ces acteurs se sont donnés les moyens de saisir les groupes au plus près de leurs comportements. À partir du cas des homosexuels masculins, l'article analyse deux innovations récentes qui incarnent cette tendance : la démédicalisation du dépistage et la prophylaxie préexposition. Celles-ci permettent de montrer que l'évolution observée ne se résume pas à l'explicitation de ce qui devait être passé sous silence, mais suppose un travail d'objectivation renouvelé, de mobilisation d'individus appartenant à la population ciblée et d'une certaine conception de l'individu preneur de risques. Le repérage des comportements à risque permet aussi de délimiter les populations sur lesquelles il est possible d'intervenir et la mise en place d'outils pour y parvenir.

\section{Mathieu Trachman, Maud Gelly, Gabriel GiRARD • Undoing and RedoIng AN At-Risk Group: The Objectivation and Prevention of Aids Among Male HomoseXuALs IN tHe ANTIRETROVIRAL ERA}

Policies on the fight against HIV and AIDS have changed considerably in France since the 2000s. In the 1990s, the fight against HIV became a national cause, the preventive initiatives of which addressed the entire population to avoid any stigmatization. In the 2000 s, recognizing the existence of "high-risk groups" no longer posed a problem and determined more targeted action. In response to the resurgence in risk-taking, the players involved in these efforts worked to understand groups through the close observation of their behaviour. Focusing on male homosexuals, the article analyses two recent innovations embodying this trend: the demedicalization of screening and pre-exposure prophylaxis. These developments show that the observed changes are not simply about expressing what should not be said, but instead involve renewed work on objectivation, the mobilization of members of the target population, and a certain idea of the risk-taking individual. The detection of at-risk behaviour also serves to delimit the populations with which intervention is possible and the implementation of the necessary tools.

\section{Mathieu Trachman, Maud Gelly, Gabriel GIRARD • DeShACER Y REHACER UN GRUPO DE RIESGO. OBJETIVACIÓN Y PREVENCIÓN DEL SIDA ENTRE LOS HOMOSEXUALES MASCULINOS EN LA ERA DE LOS ANTIRRETROVIRALES.}

Durante os años 1990, la lucha contra el VIH en Francia se ha convertido en una causa nacional y las acciones de prevención se dirigen al conjunto de la población para evitar toda discriminación. En los años 2000, el reconocimiento de la existencia de "grupos de riesgo" ya no ha planteado ningún problema y se han podido determinar acciones más selectivas. Ante la recrudescencia de las asunciones de riesgo, se ha sentido la necesidad de aprehender los grupos por una observación precisa de sus comportamientos. A partir del caso de homosexuales masculinos, este artículo analiza dos innovaciones recientes: la desmedicalización de la detección et la profilaxis anterior a la exposición. Las dos permiten mostrar que la evolución observada no se limita a la explicitación de lo que habría tenido que haber sido silenciado, sino que supone un trabajo de objetivación renovado, de movilización de individuos pertenecientes a la población considerada y de una cierta concepción del individuo que asume riesgos. La identificación de comportamientos de riesgo permite también delimitar las poblaciones sobre las que es posible intervenir y establecer los instrumentos para lograrlo.

Mots-clés : VIH/sida, homosexualité masculine, prévention, dépistage, prophylaxie, France

Keywords: HIV/AIDS, male homosexuality, prevention, screening, prophylaxis, at-risk, PrEP, France 